\title{
The JAK/STAT pathway is involved in the upregulation of PD-L1 expression in pancreatic cancer cell lines
}

\author{
TOSHIFUMI DOI ${ }^{1}$, TAKESHI ISHIKAWA ${ }^{1,2}$, TETSUYA OKAYAMA ${ }^{1,2}$, KANAME OKA $^{1}$, KATSURA MIZUSHIMA ${ }^{1}$, \\ TOMOYO YASUDA $^{1}$, NAOYUKI SAKAMOTO ${ }^{1}$, KAZUHIRO KATADA $^{1}$, KAZUHIRO KAMADA $^{1}$, \\ KAZUHIKO UCHIYAMA ${ }^{1}$, OSAMU HANDA ${ }^{1}$, TOMOHISA TAKAGI ${ }^{1}$, \\ YUJI NAITO $^{1}$ and YOSHITO ITOH ${ }^{1}$
}

\author{
Departments of ${ }^{1}$ Gastroenterology and Hepatology, and ${ }^{2}$ Cancer ImmunoCell Regulation, \\ Graduate School of Medical Science, Kyoto Prefectural University of Medicine, \\ Kamigyo-ku, Kyoto 602-8566, Japan
}

Received March 28, 2016; Accepted December 28, 2016

DOI: 10.3892/or.2017.5399

\begin{abstract}
Although improvements in the chemotherapy modalities for pancreatic cancer have been realized, pancreatic cancer remains one of the most lethal malignancies. New-generation cancer immunotherapy methods, such as blocking of the PD-1/PD-L1 pathway, are consistently being investigated to improve the survival of pancreatic cancer patients. In the present study, we evaluated the influence of anticancer agents 5-fluorouracil, gemcitabine and paclitaxel on PD-L1 expression in human pancreatic cancer cell lines MIA PaCa-2 and AsPC-1 and in murine pancreatic cancer cell line Pan02. Additionally, we analyzed the molecular mechanisms that facilitated the regulation of PD-L1 expression in these cell lines. We observed that when AsPC-1, MIA PaCa-2 and Pan 02 cells were stimulated by 5 -fluorouracil, gemcitabine or paclitaxel, PD-L1 surface protein expression was enhanced. Similarly, the mRNA level of PD-L1 was upregulated in the AsPC-1 and Pan02 cells when stimulated by each of the three anticancer agents. The phosphorylation of STAT1 and an increase in total STAT1 were also observed in the AsPC-1 cells when stimulated by each anticancer agent. In response to JAK2 inhibitor treatment, PD-L1 upregulation induced by the anticancer agents was reduced in a dose-dependent manner. These results suggest that i) the JAK2/STAT1 pathway is
\end{abstract}

Correspondence to: Dr Takeshi Ishikawa, Department of Gastroenterology and Hepatology, Graduate School of Medical Science, Kyoto Prefectural University of Medicine, 465 Kajii-cho, Kawaramachi-dori Hirokoji, Kamigyo-ku, Kyoto 602-8566, Japan E-mail: iskw-t@koto.kpu-m.ac.jp

Abbreviations: PD-1, programmed death-1; PD-L1, programmed death-ligand 1; JAK, Janus activated kinase; STAT, signal transducer and activator of transcription; GEM, gemcitabine; 5-FU, 5-fluorouracil; PTX, paclitaxel

Key words: programmed death-1, anticancer agents, pancreatic cancer, Janus activated kinase/signal transducer and activator of transcription pathway, cancer immunotherapy involved in the anticancer agent-mediated PD-L1 transcription; and ii) the anticancer agents altered the tumor immune response which may induce tumor immune escape. These findings can have an influence on the design of treatments that combine chemotherapy and immunotherapy.

\section{Introduction}

Pancreatic cancer is the fourth leading cause of cancer-related deaths in Japan (1) and the USA (2). This cancer is highly resistant to systemic therapies and this is partially the reason for its high mortality rate $(3,4)$. Gemcitabine chemotherapy has been the standard treatment for pancreatic cancer for about a decade; however, gemcitabine plus nanoparticle albumin-bound (nab)-paclitaxel and FOLFIRINOX (5-fluorouracil, oxaliplatin, irinotecan and leucovorin) were found to result in better overall survival (OS) and is now commonly used $(5,6)$. The drawback however is that these regimens are more toxic than gemcitabine monotherapy. Moreover, despite the improvements in chemotherapy, OS is still less than one year. Therefore, more effective and better-tolerated treatment options are required to improve the outcome for patients with advanced pancreatic cancer.

Exciting progress has been made in cancer immunotherapy with breakthrough immune checkpoint inhibitors and new cell therapies that enhance the effectiveness of T cells (7). Programmed death-1 (PD-1) found on activated T cells is a member of the CD28 family, and is a key immune checkpoint molecule. When PD-1 binds to programmed death-ligand 1 (PD-L1) or PD-L2, the T cell receives an inhibitory signal and no longer mounts productive immune responses $(8,9)$. Nivolumab, a fully human IgG4 PD-1 antibody, restores anticancer immune responses by abrogating PD-1 pathway-mediated $\mathrm{T}$ cell inhibition and has been approved for use in Japan and the USA for treating patients with unresectable melanoma $(9,10)$. The clinical efficacy of nivolumab has also been reported in patients with non-small cell lung cancer (11) and renal cell carcinoma (12). In addition to PD-1 and PD-L1, other immune checkpoint inhibitors are currently being developed for various tumors $(13,14)$. 
PD-1 or PD-L1 antibodies have been shown to exert a substantial antitumor effect in mouse pancreatic cancer models $(15,16)$. Although no studies to date have demonstrated the clinical efficacy of immune checkpoint inhibitor monotherapy for pancreatic cancer $(17,18)$, ipilimumab (a checkpoint inhibitor of CTLA-4) combined with cancer vaccine has shown a survival benefit $(19,20)$. Thus, immune checkpoint inhibitors combined with immune- or non-immune-based therapies are great prospective treatment strategies for pancreatic cancer.

It is still unclear what impact anticancer agents have on immune checkpoint molecules when chemotherapy is combined with immunotherapy. Very few studies have addressed the PD-1/PD-L1 pathways, and there are contradictions among them (21-24). It is within this context that we investigated how anticancer agents influence PD-L1 expression in pancreatic cancer cell lines. In the present study, we demonstrated that commonly used anticancer agents for pancreatic cancer (i.e. gemcitabine, 5-fluorouracil and paclitaxel) upregulate cell surface PD-L1 expression in both human and mouse pancreatic cancer cell lines. Additionally, we provide evidence that not only the MAPK and PI3K/AKT pathway, which are known pathways, but also the JAK/STAT pathway is involved in the induction of PD-L1 expression in response to these anticancer agents.

\section{Materials and methods}

Cell lines and reagents. The human pancreatic cancer cell line MIA PaCa-2 was obtained from Riken BioResource Center (Tsukuba, Japan) and AsPC-1 cells were obtained from the American Type Culture Collection (ATCC; Rockville, MD, USA). The murine pancreatic cancer cell line Pan02, which is syngeneic to $\mathrm{C} 57 \mathrm{Bl} / 6$ mice, was purchased from the Division of Cancer Treatment and Diagnosis, National Cancer Institute (Bethesda, MD, USA). AsPC-1 and Pan02 cells were grown in $75 \mathrm{~cm}^{2}$ cell culture flasks and maintained in Roswell Park Memorial Institute (RPMI)-1640 medium supplemented with $10 \%$ fetal bovine serum (FBS), L-glutamine and penicillin $(100 \mathrm{U} / \mathrm{ml}) / \mathrm{streptomycin}(100 \mu \mathrm{g} / \mathrm{ml})$ (both from Gibco Life Technologies, Carlsbad, CA, USA). MIA PaCa-2 cells were cultured in Dulbecco's modified Eagle's medium low glucose supplemented with $10 \% \mathrm{FBS}$, L-glutamine and penicillin $(100 \mathrm{U} / \mathrm{ml}) /$ streptomycin $(2.5 \mu \mathrm{g} / \mathrm{ml})$. Cells were incubated at $37^{\circ} \mathrm{C}$ in a humidified atmosphere containing $5 \% \mathrm{CO}_{2}$.

In the present study, we used gemcitabine (GEM), 5-fluorouracil (5-FU) and paclitaxel (PTX) which are agents commonly used to treat pancreatic cancer; all agents were immediately prepared before use. GEM was purchased from the Tokyo Chemical Industry Co., Ltd. (Tokyo, Japan), 5-FU from Kyowa Hakko Kirin Co., Ltd. (Tokyo, Japan) and PTX was obtained from Nippon Kayaku Co., Ltd. (Tokyo, Japan).

Evaluation of PD-L1 expression after exposure to anticancer

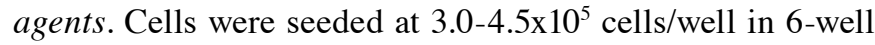
plates $48 \mathrm{~h}$ before treatment and left to incubate at $37^{\circ} \mathrm{C}$ in a humidified atmosphere containing $5 \% \mathrm{CO}_{2}$. After $48 \mathrm{~h}$, cells were exposed to various concentrations of GEM, 5-FU and PTX for 6-72 h. For each drug, the concentration used in our experiment was based on their plasma level in clinical use. The expression level of PD-L1 was determined using flow cytometry and qRT-PCR.
Treatment with JAK/STAT and other pathway inhibitors. AsPC-1 cells were adjusted to $4.5 \times 10^{5}$ cells/well into 6 -well plates. After a 48 -h incubation at $37^{\circ} \mathrm{C}$ in a humidified atmosphere containing $5 \% \mathrm{CO}_{2}$, the cells were treated for $1 \mathrm{~h}$ with various concentrations of JAK2 (AG490; Santa Cruz Biotechnology, Inc., Dallas, TX, USA), Akt (perifosine) or MEK1/2 inhibitors (U012) (both from Cell Signaling Technology, Inc., Danvers, MA, USA). Cells were stimulated with 5-FU, PTX and GEM and then incubated in the presence or absence of AG490 for an additional 6-48 h. PD-L1 expression was analyzed using flow cytometry and qRT-PCR.

Western blotting. Cells were washed twice with phosphate-buffered saline (PBS) and lysed in ice-cold lysis buffer (CelLytic $^{\mathrm{TM}}$ MT cell lysis reagent) containing 2\% proteinase inhibitor (both from Sigma-Aldrich Co., St. Louis, MO, USA). Cells were retrieved with a cell scraper, stirred and incubated on ice for $15 \mathrm{~min}$. Lysates were centrifuged, supernatants were collected, and protein concentration was determined using the Bio-Rad protein assay (Bio-Rad Laboratories, Hercules, CA, USA). The supernatants were diluted with NuPAGE LDS sample buffer (Life Technologies, Grand Island, NY, USA) to create equal concentrations of protein. Ten micrograms of protein were separated on 4-12\% NuPage Bis-Tris gels and blotted onto a nitrocellulose membrane using the iBlot Dry Blotting System (all from Life Technologies) according to the manufacturer's protocol. Blots were blocked with $10 \%$ EzBlock Chemi (ATTO Corporation, Tokyo, Japan) in TBS-T [10 mM Tris-HCl (pH 8.0), $150 \mathrm{mM} \mathrm{NaCl}, 0.1 \%$ Tween-20 v/v] for $1 \mathrm{~h}$ at room temperature and washed with TBS-T 3 times. The membranes were incubated overnight at $4^{\circ} \mathrm{C}$ with anti-STAT1, anti-phospho-STAT1, anti-NF- $\kappa$ B (all from Cell Signaling Technology, Inc.), anti-Akt1/2/3, anti-phospho-Akt1/2/3, anti-p38, anti-phospho-p38 (all from Santa Cruz Biotechnology, Inc.), anti-JNK (Cell Signaling Technology, Inc.), anti-phospho-JNK (Santa Cruz Biotechnology, Inc.), anti-Erk 1/2 (Cell Signaling Technology, Inc.), anti-phosphoErk 1/2, anti-tubulin (both from Santa Cruz Biotechnology, Inc.) and anti- $\beta$ actin (Abcam, Cambridge, MA, USA) antibodies in TBS-T (diluted 1:1,000). After washing in TBS-T, the membranes were incubated with the secondary anti-rabbit and mouse IgG antibodies (Life Technologies) in TBS-T (diluted $1: 10,000)$ for $1 \mathrm{~h}$ at room temperature. Immunocomplexes were detected using western blotting (ECL Prime; GE Healthcare UK Ltd., Buckinghamshire, UK).

Quantitative reverse transcription-polymerase chain reaction ( $q R T-P C R)$. The expression level of PD-L1 was determined using real-time PCR as previously described (25). The samples used for mRNA isolation were removed from the pancreatic cancer cells (AsPC-1, MIA PaCa-2 and Pan02). Total mRNA was extracted using the acid guanidinium phenol chloroform method with Isogen (Nippon Gen Co. Ltd., Tokyo, Japan). The isolated RNA was stored at $-80^{\circ} \mathrm{C}$ until use for real-time PCR. In the latter, $1 \mu \mathrm{g}$ of extracted RNA was reverse-transcribed. The resulting cDNA was subjected to qRT-PCR using the following primers for human PD-L1: (forward primer, 5'-GTACCGCTGCATGATCAGCTAT-3' and reverse primer, 5'-GGCATTGACTTTCACAGTAATTCG-3'); murine PD-L1 (forward primer, 5'-CAGGCCGAGGGTTATCCA-3' and 
A AsPC-1
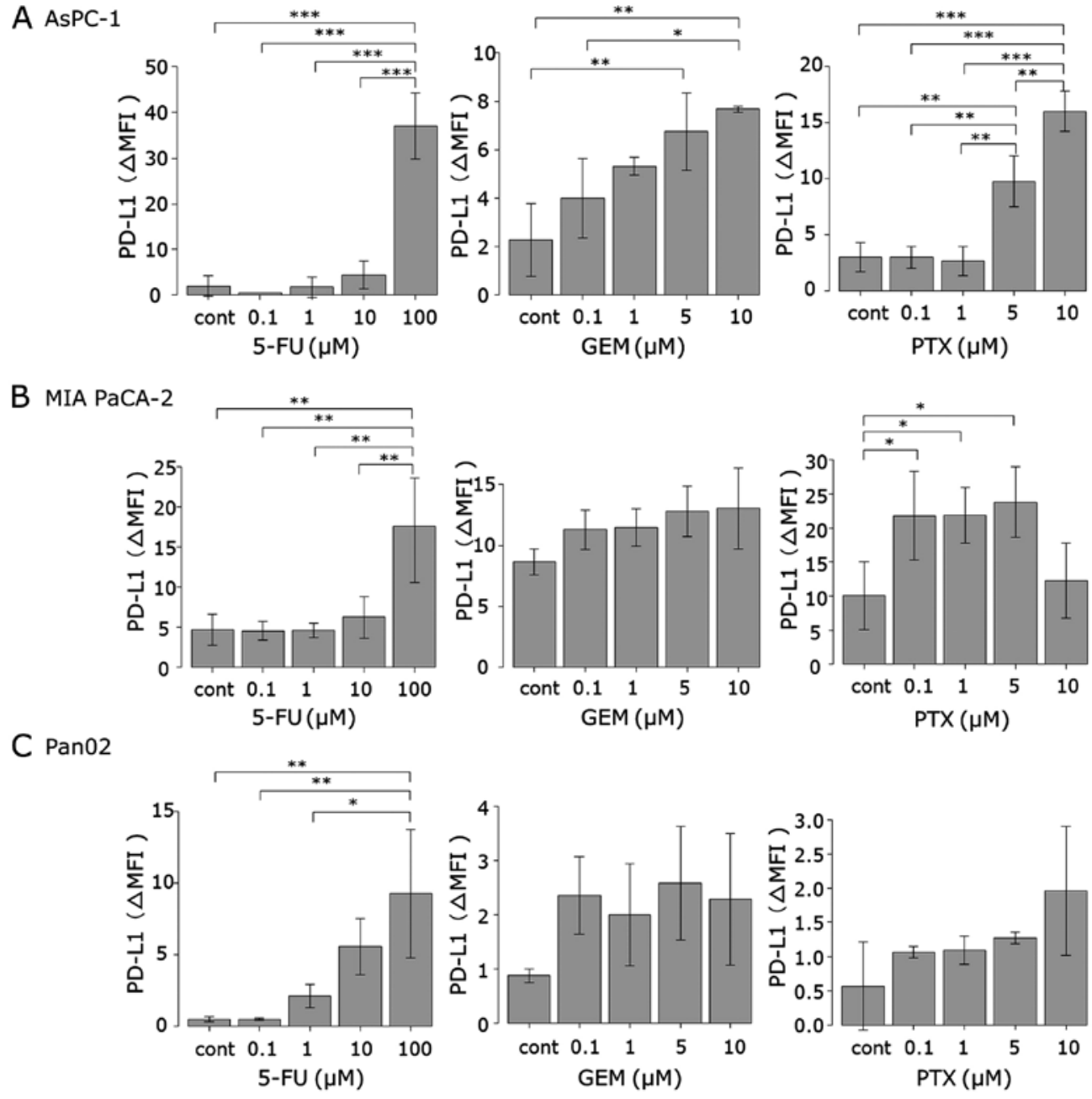

Figure 1. PD-L1 surface protein expression in pancreatic cancer cell lines. (A) AsPC-1, (B) MIA PaCa-2 and (C) Pan02 cells were stimulated by three anticancer agents (5-FU for $72 \mathrm{~h}$, GEM for $24 \mathrm{~h}$ and PTX for $48 \mathrm{~h}$ ). PD-L1 expression was then analyzed using flow cytometry, and is presented as $\triangle$ MFI (MFI using anti-PD-L1 subtracted from the isotype control). Data represent the mean \pm standard error of the mean (SEM) of at least 3 independent experiments; ${ }^{*} \mathrm{P}<0.05,{ }^{* *} \mathrm{P}<0.01,{ }^{* * * *} \mathrm{P}<0.001$. Comparisons among groups were evaluated by one-way ANOVA and Tukey-Kramer multiple comparison test. 5-FU, 5-fluorouracil; GEM, gemcitabine; PTX, paclitaxel.

reverse primer, 5'-CGGGTTGGTGGTCACTGTTT-3'); human GAPDH (forward primer, 5'-ACCACAGTCCATGCC ATCACT-3' and reverse primer, CCATCACGCCACAGTTT CC); and murine $\beta$-actin (forward primer, 5 '-TATCCACCTTC CAGCAGATGT-3' and reverse primer, 5'-AGCTCAGTAAC AGTCCGCCTA-3'). PCR was performed using a Power SYBR-Green PCR Master Mix and a real-time PCR system (7300; Applied Biosystems, Foster City, CA, USA). Relative quantifications of gene expression with qRT-PCR data were calculated relative to human GAPDH or murine $\beta$-actin.

Flow cytometric analysis. PD-L1 surface expression was analyzed by flow cytometry. Cells harvested from in vitro cultures were washed twice with CellWash ${ }^{\text {TM }}$ (Becton-Dickinson and Co., Franklin Lakes, NJ, USA) and then incubated with anti-PD-L1 (human, Becton-Dickinson and Co., mouse, eBioscience, San Diego, CA, USA); or isotype control antibodies (eBioscience) for $30 \mathrm{~min}$ at $4^{\circ} \mathrm{C}$. The cells were washed with CellWash once and analyzed by flow cytometer on a FACSCalibur flow cytometer and CellQuest ${ }^{\mathrm{TM}}$ Pro version 6.0 software (both from Becton-Dickinson and Co.).
Statistical analysis. Results are expressed as means \pm standard error of the mean (SEM). Comparisons among groups or against one control group were evaluated by one-way ANOVA test followed by the Tukey-Kramer's or Dunnett's post hoc multiple comparisons test, respectively. Statistical significance was taken as $\mathrm{P}<0.05$. Statistical analyses were performed with EZR (Saitama Medical Center, Jichi Medical University, Saitama, Japan), which is a graphical user interface for $\mathrm{R}$ (The $\mathrm{R}$ Foundation for Statistical Computing, Vienna, Austria). More precisely, it is a modified version of $\mathrm{R}$ commander designed to add statistical functions frequently used in biostatistics (26).

\section{Results}

Anticancer agents upregulate PD-L1 surface expression in pancreatic cancer cell lines. MIA PaCa-2, AsPC-1 and Pan02 cells were treated with GEM, 5-FU and PTX for 24-72 h to determine whether they can induce PD-L1 protein expression. Expression was determined using flow cytometry and is expressed as the $\Delta$ mean fluorescence intensity ( $\Delta \mathrm{MFI}$; MFI using anti-PD-L1 subtracted from the isotype control). As 
A AsPC-1
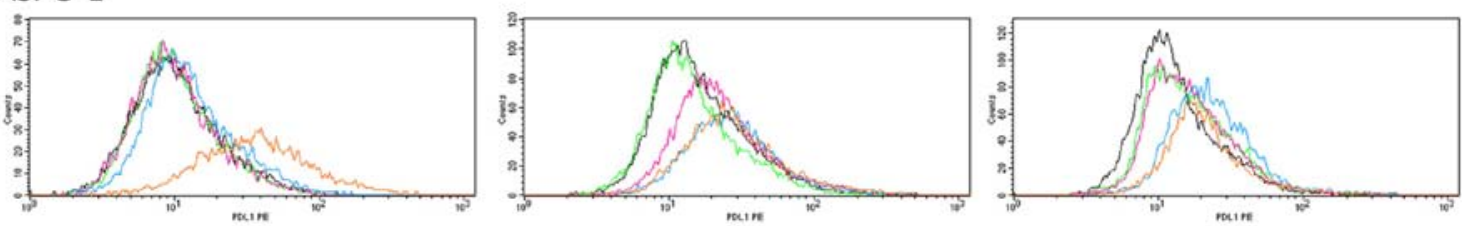

B MIA PaCA-2
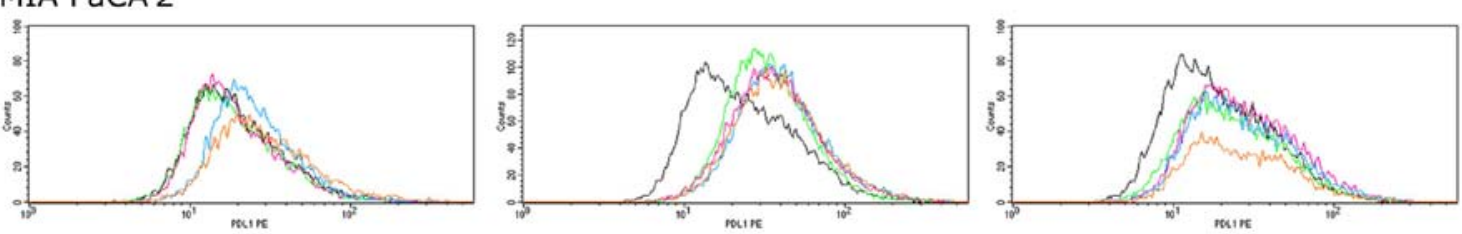

C Pan02

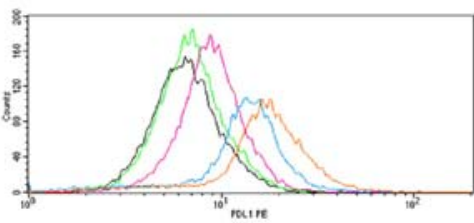

5-FU

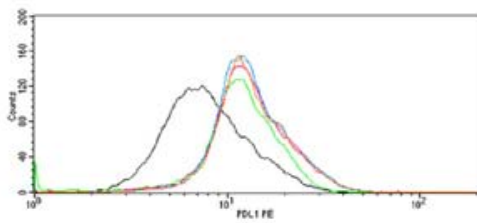

GEM

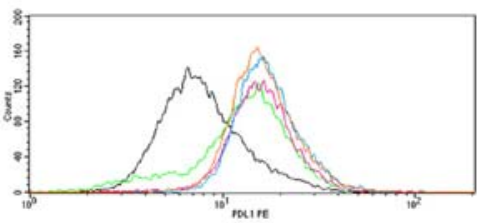

PTX

Figure 2. PD-L1 surface protein expression in pancreatic cancer cell lines. (A) AsPC-1, (B) MIA PaCa-2 and (C) Pan02 cells were stimulated by three anticancer agents (5-FU for $72 \mathrm{~h}$, GEM for $24 \mathrm{~h}$ and PTX for $48 \mathrm{~h}$ ). PD-L1 expression was then analyzed using flow cytometry. The concentrations of each anticancer agents are indicated as follows: 5-FU: black, $0 \mu \mathrm{M}$; green, $0.1 \mu \mathrm{M}$; pink, $1 \mu \mathrm{M}$; blue, $10 \mu \mathrm{M}$; orange, $100 \mu \mathrm{M}$. GEM and PTX: black, $0 \mu \mathrm{M}$; green, $0.1 \mu \mathrm{M}$; pink, $1 \mu \mathrm{M}$; blue, $5 \mu \mathrm{M}$; orange, $10 \mu \mathrm{M}$. 5-FU, 5-fluorouracil; GEM, gemcitabine; PTX, paclitaxel.

shown in Figs. 1 and 2, treatment with 5-FU for $72 \mathrm{~h}$ induced PD-L1 surface expression in both human pancreatic cancer cell lines (MIA PaCa-2 and AsPC-1) at $100 \mu \mathrm{M}$, whereas 5-FU did not affect the expression at concentrations $<100 \mu \mathrm{M}$. In mouse pancreatic cancer cell line Pan02, 5-FU (72 h) at concentrations $>1 \mu \mathrm{M}$ induced PD-L1 expression in a dosedependent manner. Treatment with GEM for $24 \mathrm{~h}$ induced PD-L1 expression in the AsPC-1 cells in a dose-dependent manner. In both MIA PaCa-2 and Pan02 cell lines, GEM induced PD-L1 expression at each concentration, but it did not reach statistical significance. Treatment with PTX for $48 \mathrm{~h}$ significantly induced PD-L1 expression in the AsPC-1 cells at concentrations of 5 and $10 \mu \mathrm{M}$ and in MIA PaCa-2 cells at each concentration except $10 \mu \mathrm{M}$. PTX also induced PD-L1 expression in mouse Pan02 cells at each concentration, but it did not reach statistical significance.

Anticancer agents induce PD-L1 mRNA expression in pancreatic cancer cell lines. To investigate how the anticancer agents induce PD-L1 protein expression in tumor cells, the mRNA level of PD-L1 was determined by qRT-PCR. MIA PaCa-2, AsPC-1 and Pan02 cells were treated with 5-FU, GEM and PTX for $24 \mathrm{~h}$ in a manner similar as that carried out for the protein assessment as described above. In AsPC-1 cells, 5-FU significantly increased the PD-L1 mRNA level at $100 \mu \mathrm{M}$, as observed in the surface protein expression. Treatment with GEM at 5 and $10 \mu \mathrm{M}$ significantly upregulated mRNA expression while each dose of PTX upregulated mRNA expression although not to statistically significant levels (Fig. 3A). In the MIA PaCa-2 cells, neither 5-FU nor GEM affected PD-L1 mRNA expression, whereas PTX at $10 \mu \mathrm{M}$ significantly increased the mRNA level (Fig. 3B). The PD-L1 mRNA expression pattern in the Pan02 cells was similar to that of the surface protein, that is, the mRNA level increased when 5-FU exceeded $1 \mu \mathrm{M}$, while GEM and PTX upregulated the mRNA level at each concentration (Fig. 3C).

Anticancer agents activate the JAK/STAT pathway. The protein and mRNA expression analyses suggest that surface PD-L1 protein expression in the AsPC-1 and Pan02 cells was regulated mainly at the mRNA level. As such, we used the human AsPC-1 cell line to ascertain the molecular mechanisms by which the anticancer agents induced PD-L1 mRNA expression. It has been reported that the JAK/STAT pathway is deeply involved in IFN- $\gamma$-mediated PD-L1 upregulation in solid tumors such as lung cancer and hepatocellular carcinoma $(27,28)$. In contrast, this pathway has not been implicated in anticancer agent-mediated PD-L1 expression. As such, we aimed to ascertain whether our three anticancer agents activate the JAK/STAT signaling pathway in AsPC-1 cells. AsPC-1 cells were exposed to 5-FU (100 $\mu \mathrm{M})$ or GEM $(10 \mu \mathrm{M})$ for $48 \mathrm{~h}$, and PTX $(10 \mu \mathrm{M})$ for $24 \mathrm{~h}$ following which the expression level and phosphorylation of STAT1 were determined using western blotting. The phosphorylation of STAT1 was greatly induced by each of the three anticancer agents (Fig. 4). The protein level of STAT1 was increased after 5-FU and GEM treatment, whereas it remained unchanged after PTX treatment.

Anticancer agent-induced PD-L1 expression is attenuated by blocking the JAK/STAT pathway. Next, we examined the effect of a JAK 2 inhibitor on the upregulation of PD-L1 expression induced by the anticancer agents in AsPC-1 cells. Cells were treated with the JAK2 inhibitor AG490 or left untreated prior 

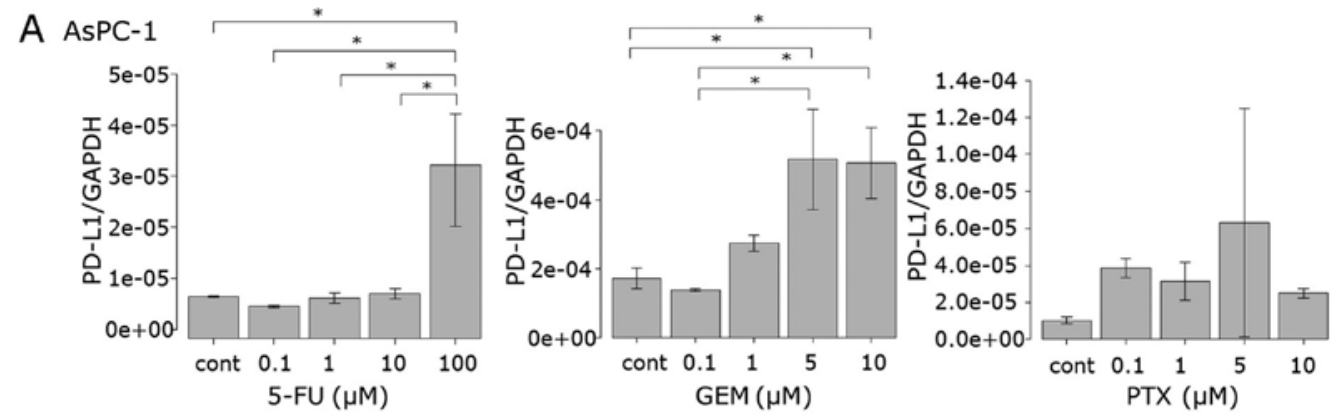

B MIA PaCA-2
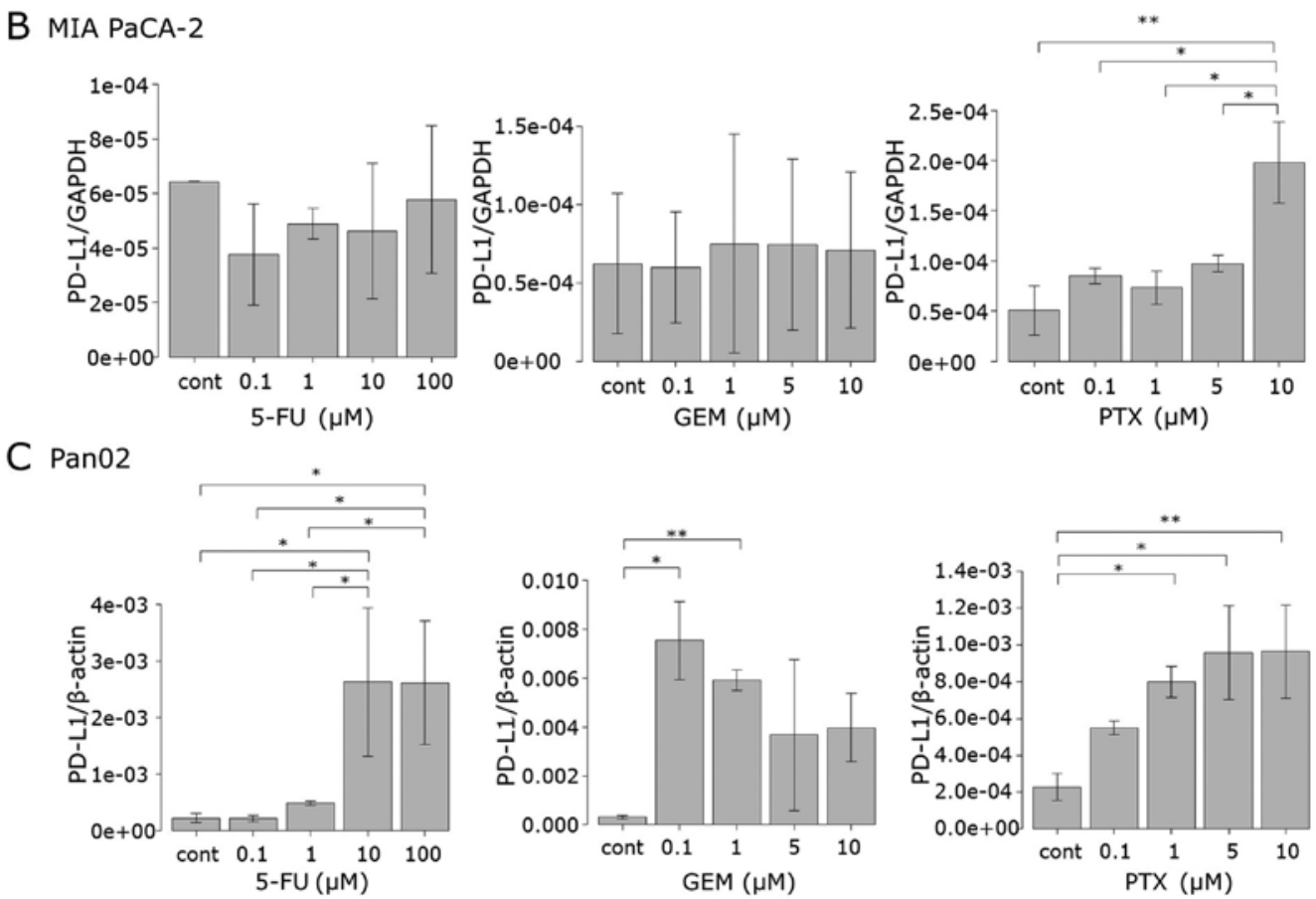

Figure 3. PD-L1 mRNA expression in pancreatic cancer cell lines. (A) AsPC-1, (B) MIA PaCa-2 and (C) Pan02 cells were stimulated by 5-FU, GEM and PTX for $24 \mathrm{~h}$. PD-L1 mRNA expression was then analyzed using qRT-PCR. Relative gene expression for qRT-PCR data was calculated relative to human GAPDH or murine $\beta$-actin. Comparisons among groups were evaluated by one-way ANOVA and Tukey-Kramer multiple comparison test. Data represent the mean \pm standard error of the mean (SEM) of at least 3 independent experiments; ${ }^{*} \mathrm{P}<0.05,{ }^{* *} \mathrm{P}<0.01$. 5-FU, 5-fluorouracil; GEM, gemcitabine; PTX, paclitaxel.
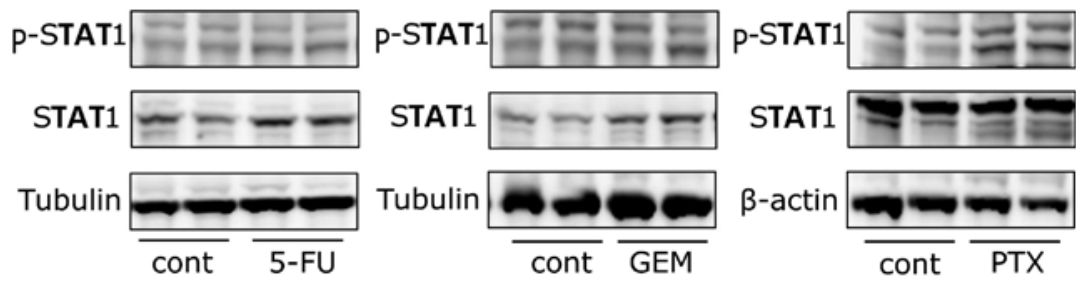

Figure 4. STAT1 phosphorylation in AsPC-1 cells. AsPC-1 cells were cultured in the presence or absence of 5-FU, GEM and PTX for 24-48 h, and then phospho-STAT1 and STAT1 protein levels were analyzed using western blotting. 5-FU, 5-fluorouracil; GEM, gemcitabine; PTX, paclitaxel.

to 5-FU, PTX or GEM stimulation. Both PD-L1 mRNA expression (Fig. 5A) and cell surface protein (Fig. 5B) induced by the anticancer agents were attenuated by AG490 in a dosedependent manner. AG490 at $100 \mu \mathrm{M}$ almost completely attenuated the PD-L1 expression induced by the anticancer agents.

Involvement of other pathways. Additionally, we also investigated whether the PI3K/AKT (29), NF- $\mathrm{B}(24,30)$ and
MAPK $(23,31)$ pathways respond to anticancer agents, since they were previously implicated in anticancer agent-induced PD-L1 expression. The phosphorylation of Akt was induced by only GEM stimulation, although its protein level was enhanced by 5-FU and GEM (Fig. 6). The protein level of Erk was enhanced by each of the three anticancer agents. The intracellular protein level of $N F-\kappa B$ and the phosphorylation and protein levels of p38 were not affected by the drugs (Fig. 6). Considering the results of the western blot 

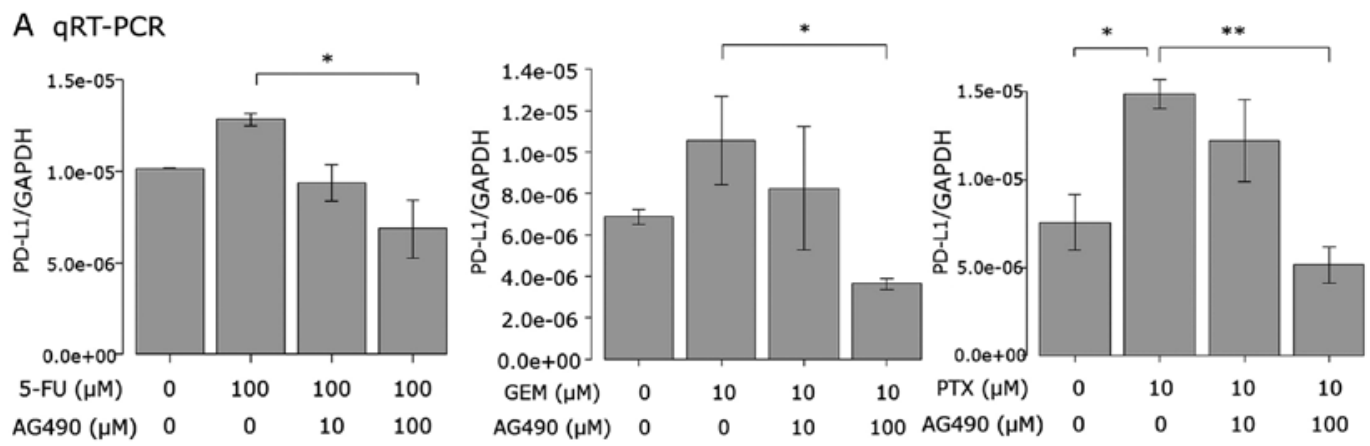

B Flow cytometry
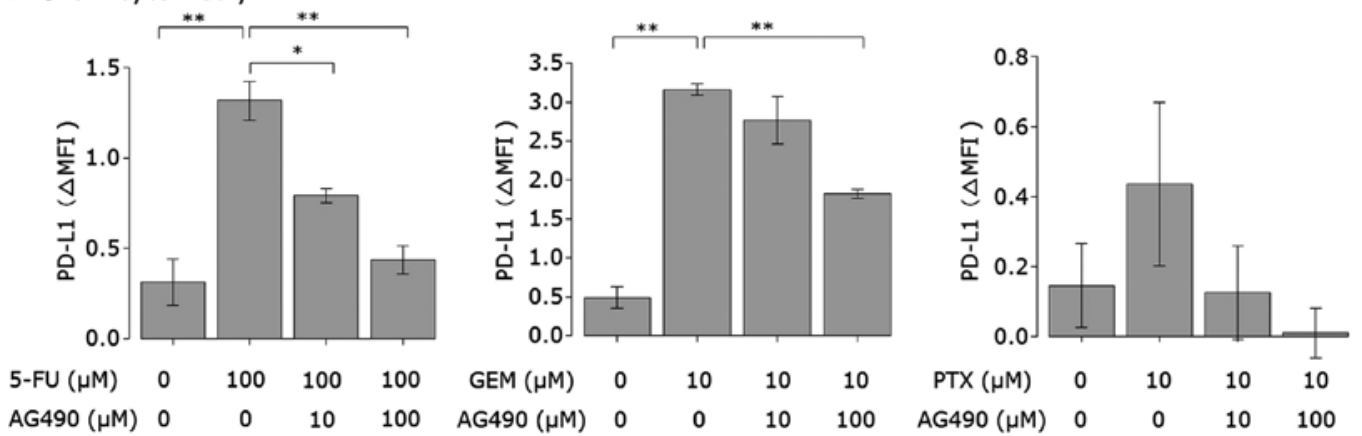

Figure 5. Effect of the JAK2 inhibitor on PD-L1 expression in pancreatic cancer cells. AsPC-1 cells were pre-treated with 0, 10 or $100 \mu \mathrm{M}$ of AG490 (the JAK2 inhibitor), and then stimulated with 5-FU, GEM and PTX. (A) PD-L1 mRNA expression was analyzed using qRT-PCR after $6 \mathrm{~h}$. Relative quantification of gene expression for the qRT-PCR data was calculated relative to GAPDH. (B) PD-L1 surface protein expression was analyzed using flow cytometry, and is presented as $\triangle$ MFI (MFI using anti-PD-L1 subtracted from the isotype control). Comparisons among groups were evaluated by one-way ANOVA test followed by Dunnett comparisons test (vs. anticancer agents and AG490 $0 \mu \mathrm{M}$ group). Data represent the mean \pm standard error of the mean (SEM) of 2 independent experiments; ${ }^{*} \mathrm{P}<0.05,{ }^{* *} \mathrm{P}<0.01$. 5-FU, 5-fluorouracil; GEM, gemcitabine; PTX, paclitaxel.

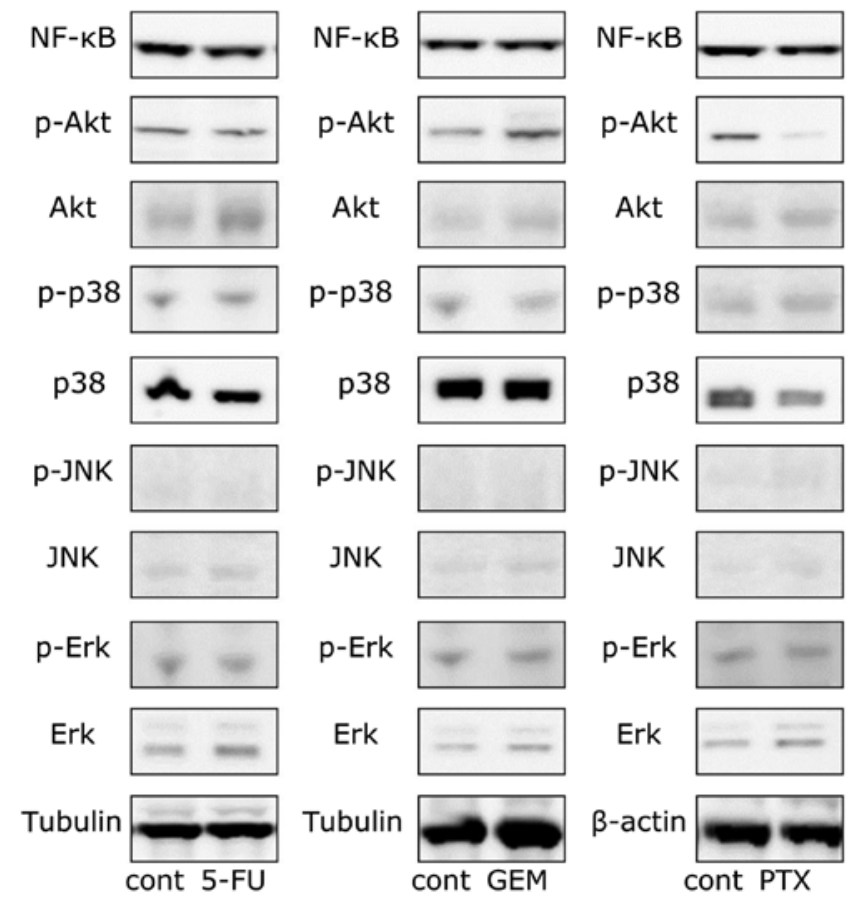

Figure 6. Effect of the anticancer agents on PI3K/AKT, NF- $\mathrm{kB}$ and MAPK in AsPC-1 cells. AsPC-1 cells were cultured in the presence or absence of 5-FU, GEM and PTX for 24-48 h, and then phosphorylation and protein levels of Akt, p38, JNK, Erk and the intracellular NF- $\mathrm{kB}$ protein level were analyzed using western blotting. 5-FU, 5-fluorouracil; GEM, gemcitabine; PTX, paclitaxel.

analysis, we also investigated the effect of the inhibitors of the PI3K/AKT and MAPK pathways. As shown in Figs. 7 and 8, anticancer agent-induced PD-L1 upregulation was attenuated by the Akt and MEK1/2 inhibitors. 

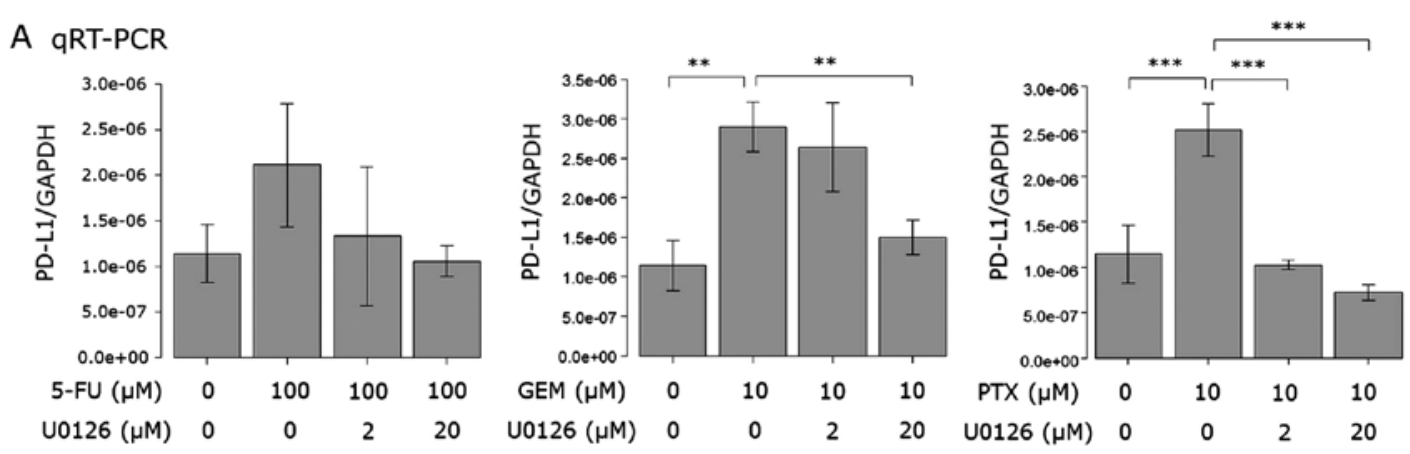

\section{B Flow cytometry}
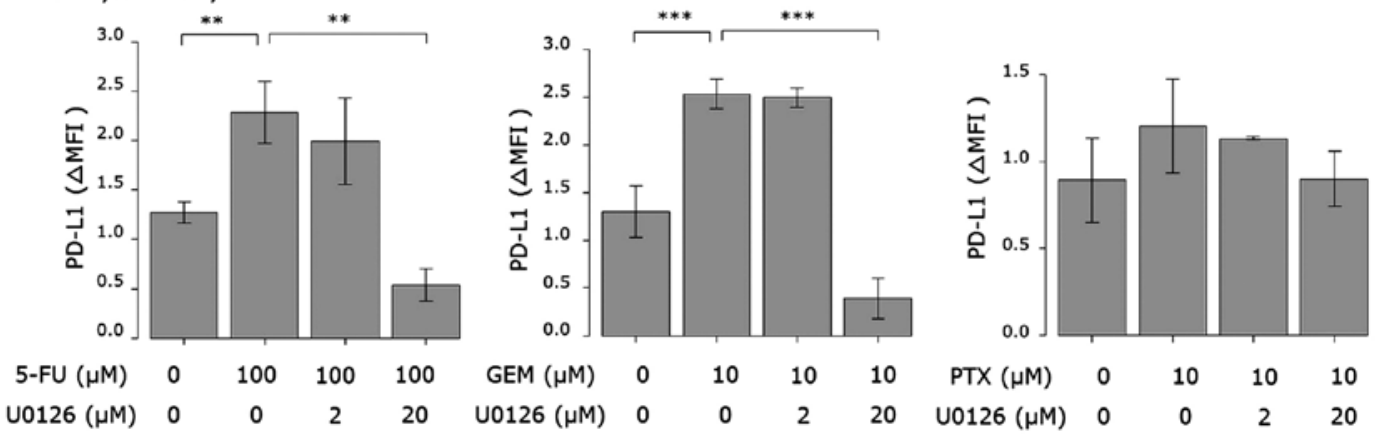

Figure 7. Effect of MEK1/2 inhibitor on PD-L1 expression in pancreatic cancer cells. AsPC-1 cells were pre-treated with 0,2 or $20 \mu \mathrm{M}$ of U0126 (an MEK1/2 inhibitor), and then stimulated with 5-FU, GEM and PTX. (A) PD-L1 mRNA expression was analyzed using qRT-PCR after $6 \mathrm{~h}$. Relative quantification of gene expression for qRT-PCR data was calculated relative to GAPDH. (B) PD-L1 surface protein expression was analyzed using flow cytometry, and is presented as MFI (MFI using anti-PD-L1 subtracted from the isotype control). Comparisons among groups were evaluated by one-way ANOVA test followed by Dunnett comparisons test (vs. anticancer agents and U0126 $0 \mu \mathrm{M}$ group). Data represent the mean \pm standard error of the mean (SEM) of 3 independent experiments; ${ }^{* *} \mathrm{P}<0.01,{ }^{* * * *} \mathrm{P}<0.001 .5$-FU, 5-fluorouracil; GEM, gemcitabine; PTX, paclitaxel.

\section{A qRT-PCR}

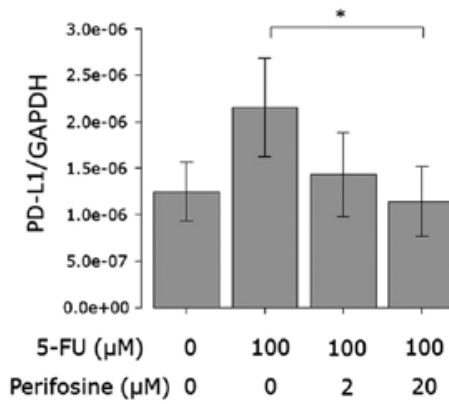

\section{B Flow cytometry}

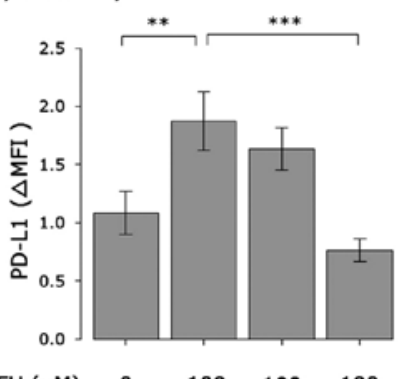

5-FU ( $\mu \mathrm{M}) \quad 0 \quad 100 \quad 100 \quad 100$

Perifosine $(\mu M) 0 \quad 0 \quad 2 \quad 20$
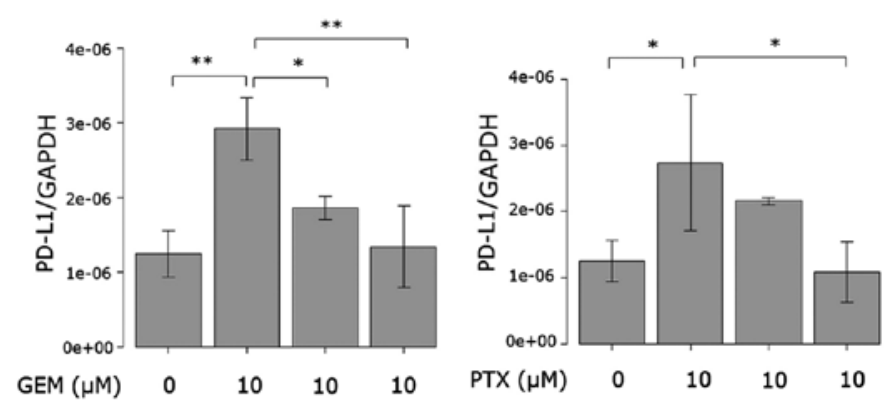

Perifosine $(\mu \mathrm{M}) 0$

20 Perifosine $(\mu M)$
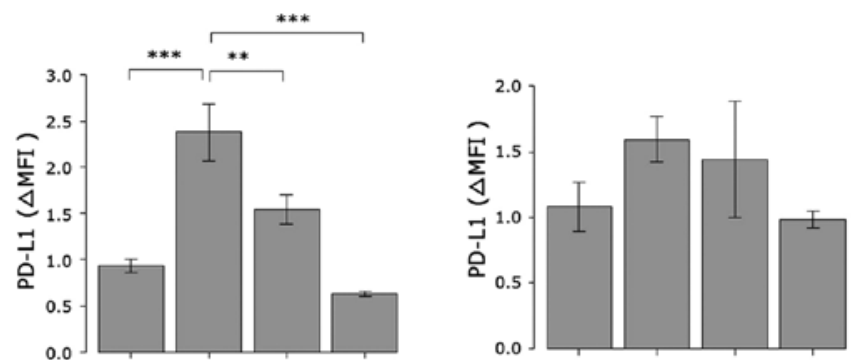

$\operatorname{GEM}(\mu \mathrm{M}) \quad 0 \quad 10 \quad 10 \quad 10 \quad \operatorname{PTX}(\mu \mathrm{M}) \quad \begin{array}{llllll}0 & 0 & 10 & 10 & 10\end{array}$

Figure 8. Effect of Akt inhibitor on PD-L1 expression in pancreatic cancer cells. AsPC-1 cells were pre-treated with 0,2 or $20 \mu \mathrm{M}$ of perifosine (an Akt inhibitor), and then stimulated with 5-FU, GEM and PTX. (A) PD-L1 mRNA expression was analyzed using qRT-PCR after 6 h. Relative quantification of gene expression for qRT-PCR data was calculated relative to GAPDH. (B) PD-L1 surface protein expression was analyzed using flow cytometry, and is presented as $\triangle$ MFI (MFI using anti-PD-L1 subtracted from the isotype control). Comparisons among groups were evaluated by one-way ANOVA test followed by Dunnett comparisons test (vs. anticancer agents and perifosine $0 \mu \mathrm{M}$ group). Data represent the mean \pm standard error of the mean (SEM) of 3 independent experiments; ${ }^{*} \mathrm{P}<0.05,{ }^{* *} \mathrm{P}<0.01,{ }^{* * *} \mathrm{P}<0.001 .5$-FU, 5-fluorouracil; GEM, gemcitabine; PTX, paclitaxel. 


\section{Discussion}

The success of immune checkpoint inhibitors and new generations of adoptive cell transfer therapy, such as chimeric antigen receptor (CAR) T cell therapy, have highlighted the potential of immunotherapy as a treatment option for various tumors $(7,32)$. Moreover, extensive effort is being directed toward effectively combining immune- and non-immunebased therapies. For example, combining immune therapy and anticancer agents is being explored, albeit the impact of anticancer agents on the expression of immune checkpoint molecules is not yet fully understood. In the present study, we present two major findings. Firstly, PD-L1 surface expression in pancreatic cancer cell lines was upregulated by 5 -fluorouracil, paclitaxel and gemcitabine. Secondly, the JAK/STAT pathway as well as other known pathways (i.e. MAPK and PI3K pathway) were involved in this PD-L1 upregulation. To the best of our knowledge, this is the first study to address the effect of anticancer agents on PD-L1 expression in pancreatic cancer cells and the involvement of the JAK/STAT pathway in the anticancer agent-mediated PD-L1 expression.

The effect of chemotherapy agents on PD-L1 expression has been discussed in four studies that we are aware of. However, there is a lack of consensus and the topic remains controversial (21-24). Among them, three studies demonstrated that the anticancer agents upregulated surface PD-L1 expression, while the fourth reported the downregulation of surface PD-L1. In their study, Zhang et al observed that paclitaxel, etoposide and 5-fluorouracil induced PD-L1 surface expression in human breast cancer cell lines (21), however they did not study the molecular mechanisms that led to the increase in PD-L1 expression. Gong et al reported that paclitaxel induced PD-L1 surface protein and mRNA expression in both human colorectal (SW480) and human hepatocellular carcinoma (HepG2) cell lines (23). They demonstrated that paclitaxel treatment induced Erk 1/2 phosphorylation in both cell lines and the increase in PD-L1 expression caused by paclitaxel was partially blocked by an MEK inhibitor. Peng reported that PD-L1 expression in ovarian cancer cell lines was augmented via NF- $\kappa$ B signaling by paclitaxel, gemcitabine or carboplatin treatment (24). In contrast, Ghebeh et al reported that doxorubicin downregulated the surface expression of PD-L1 in breast cancer cells and upregulated nuclear expression of PD-L1 by means of the PI3K/AKT pathway (22). One possible explanation for the difference among these four studies, as well as our own, may be attributed to differences in the cell lines and anticancer agents used in each study. In the present study, we used 5-fluorouracil, gemcitabine and paclitaxel since they are commonly used alone or combined with other agents when treating pancreatic cancer. The concentration of each anticancer agent in our experiments was based on the plasma level of each drug when clinically used $(33,34)$ or the concentration that was used in previous in vitro experiments $(21,35,36)$. Consequently, the maximum concentration of 5-fluorouracil in our experiment was 10-fold higher than that used for gemcitabine and paclitaxel. This difference in drug concentration among the anticancer agents may have influenced the degree of PD-L1 induction by the agents.

In the present study, PD-L1 surface protein expression was enhanced in all pancreatic cancer cell lines. The absolute value of PD-L1 expression determined by flow cytometry or qRT-PCR varied with each experiment; this could partly result from PD-L1 expression being affected by cell conditions. PD-L1 expression was consistently upregulated when stimulated by the anticancer agents; the pattern of this relative change was identical in each experiment. We observed that PD-L1 at the mRNA level was upregulated as well as surface protein expression when AsPC-1 or Pan02 cells were stimulated by each anticancer agent. Meanwhile, the mRNA level of PD-L1 in the MIA PaCa-2 cells did not increase when cells were treated with 5-fluorouracil, gemcitabine or lower concentrations of paclitaxel. It was reported that doxorubicin alters PD-L1 surface expression by a post-transcriptional regulation mechanism that involves the translocation of the protein from the membrane to the nucleus (22). Since anticancer agents induced PD-L1 surface protein expression without upregulating mRNA in the MIA PaCa- 2 cells, it is possible that the expression was mainly regulated at a posttranscriptional level in this cell line.

In regards to the mechanism of PD-L1 regulation, Pardoll reported that innate and adaptive immune resistance are the two general mechanisms by which tumor cells regulate PD-L1 (37). In innate immune resistance, PD-L1 expression is driven by constitutive oncogenic signaling pathways such as the PI3K/AKT pathway (38) and STAT3 (39). In contrast, in adoptive immune resistance, several signaling pathways such as JAK/STAT $(27,28)$, PI3K (29), MAPK (29) and NF- $\mathrm{BB}(29,30)$ appear to be involved in PD-L1 expression induced by IFN $-\gamma$. Only the PI3K/AKT (22), MAPK (23) and NF- $\mathrm{BB}$ (24) pathways have been previously reported to be involved in the upregulation of PD-L1 induced by anticancer agents, but involvement of the JAK/STAT pathway has not yet been reported. To obtain a better understanding of the mechanisms involved in PD-L1 upregulation, we sought to determine whether the JAK/STAT pathway regulates PD-L1 transcription. We found that each of the three anticancer agents induced the phosphorylation of STAT1 in the AsPC-1 cells, and JAK2 inhibitor AG490 reversed the upregulation of PD-L1 induced by the anticancer agents at both the mRNA and protein levels. These findings indicate that the JAK/STAT pathway regulates the expression of PD-L1. Other previously reported signaling pathways (i.e. MAPK and PI3K pathways) are also implicated in the present study. The relative importance of these pathways is unknown at the present, but we believe that these pathways are intricately involved in the anticancer agent-mediated PD-L1 expression by signaling crosstalk.

The discoveries made in the present study are a critical step towards further uncovering the mechanism of PD-L1 expression in pancreatic cell lines albeit with a few limitations. In the present study, we examined the signaling pathways in AsPC-1 cells only, therefore it is unclear whether these results apply to other cell lines. Pancreatic cancer cell lines including AsPC-1 and MIA PaCa-2 have different genetic alterations such as the KRAS (v-Ki-ras2 Kirsten rat sarcoma viral oncogene homolog), TP53 (encoding the p53 protein) and SMAD4 (SMAD family member 4, also known as DPC4; deleted in pancreatic carcinoma locus 4) gene mutations, which affect growth characteristics, tumorigenicity and chemosensitivity (40). AsPC-1 is considered more resistant to gemcitabine than MIA PaCa-2 $(40,41)$. Gemcitabine resistance 
has been liked to signaling pathways associated with PD-L1 expression such as JAK/STAT, MAPK, PI3K-AKT and $\mathrm{NF}-\kappa \mathrm{B}(42-45)$. These genetic alterations and the difference in the cell signaling response to anticancer agents can also alter the PD-L1 expression induced by the anticancer agents. Future research using additional cell lines is needed to clarify the relationship between these genotypic differences among cell lines and their effect on the PD-L1 expression induced by anticancer agents.

In conclusion, our results indicate that 5-fluorouracil, gemcitabine and paclitaxel enhance PD-L1 expression in pancreatic cancer cell lines via several pathways including the JAK/STAT pathway. Pancreatic cancer is still intractable due to its resistance to conventional treatments including anticancer agents. Our results imply that anticancer agents not only cause cytotoxicity, but also alter the tumor immune response which may induce tumor immune escape. Cancer immunotherapy including blockade of PD-1/PD-L1 is expected to become the new standard therapy for many cancers and combination strategy in immunotherapy is currently being developed. We believe that the data provided in the present study, may aid in the design of more effective treatments that combine chemotherapy and immunotherapy.

\section{Acknowledgements}

The present study was supported by Grant-in-Aid for Scientific Research (C) to T.I. (no. 26460914) and Grant-in-Aid for Young Scientist (B) to T.O. (no. 26830112) from the Ministry of Education, Culture, Sports, Science and Technology of Japan. Yoshito Itoh received a lecture fee and is affiliated with a department that was partially funded by Bristol-Myers Squibb, and receives a grant from Kyowa Hakko Kirin Co. Ltd.

\section{References}

1. Statistics and Information Department, Ministry of Health, Labour and Welfare: Vital Statistics. Tokyo, 2013.

2. Siegel RL, Miller KD and Jemal A: Cancer statistics, 2015. CA Cancer J Clin 65: 5-29, 2015

3. Mazur PK and Siveke JT: Genetically engineered mouse models of pancreatic cancer: Unravelling tumour biology and progressing translational oncology. Gut 61: 1488-1500, 2012.

4. Dorado J, Lonardo E, Miranda-Lorenzo I and Heeschen C: Pancreatic cancer stem cells: New insights and perspectives. J Gastroenterol 46: 966-973, 2011.

5. Von Hoff DD, Ervin T, Arena FP, Chiorean EG, Infante J, Moore M, Seay T, Tjulandin SA, Ma WW, Saleh MN, et al: Increased survival in pancreatic cancer with nab-paclitaxel plus gemcitabine. N Engl J Med 369: 1691-1703, 2013.

6. Conroy T, Desseigne F, Ychou M, Bouché O, Guimbaud R, Bécouarn Y, Adenis A, Raoul JL, Gourgou-Bourgade S, de la Fouchardière $\mathrm{C}$, et al; Groupe Tumeurs Digestives of Unicancer; PRODIGE Intergroup: FOLFIRINOX versus gemcitabine for metastatic pancreatic cancer. N Engl J Med 364: $1817-1825,2011$

7. Couzin-Frankel J: Breakthrough of the year 2013. Cancer immunotherapy. Science 342: 1432-1433, 2013.

8. Chen L: Co-inhibitory molecules of the B7-CD28 family in the control of T-cell immunity. Nat Rev Immunol 4: 336-347, 2004.

9. Brahmer JR, Hammers H and Lipson EJ: Nivolumab: Targeting PD-1 to bolster antitumor immunity. Future Oncol 11: 1307-1326, 2015.

10. Larkin J, Chiarion-Sileni V, Gonzalez R, Grob JJ, Cowey CL, Lao CD, Schadendorf D, Dummer R, Smylie M, Rutkowski P, et al: Combined nivolumab and ipilimumab or monotherapy in untreated melanoma. N Engl J Med 373: 23-34, 2015.
11. Brahmer J, Reckamp KL, Baas P, Crinò L, Eberhardt WE, Poddubskaya E, Antonia S, Pluzanski A, Vokes EE, Holgado E, et al: Nivolumab versus docetaxel in advanced squamous-cell non-small-cell lung cancer. N Engl J Med 373: 123-135, 2015.

12. Motzer RJ, Rini BI, McDermott DF, Redman BG, Kuzel TM, Harrison MR, Vaishampayan UN, Drabkin HA, George S, Logan TF, et al: Nivolumab for metastatic renal cell carcinoma: Results of a randomized phase II trial. J Clin Oncol 33: 1430-1437, 2015.

13. Li X, Hu W, Zheng X, Zhang C, Du P, Zheng Z, Yang Y, Wu J, Ji M, Jiang J, et al: Emerging immune checkpoints for cancer therapy. Acta Oncol 54: 1706-1713, 2015.

14. Le Mercier I, Lines JL and Noelle RJ: Beyond CTLA-4 and $\mathrm{PD}-1$, the generation $\mathrm{Z}$ of negative checkpoint regulators. Front Immunol 6: 418, 2015.

15. Nomi T, Sho M, Akahori T, Hamada K, Kubo A, Kanehiro H, Nakamura S, Enomoto K, Yagita H, Azuma M, et al: Clinical significance and therapeutic potential of the programmed death-1 ligand/programmed death-1 pathway in human pancreatic cancer. Clin Cancer Res 13: 2151-2157, 2007.

16. Okudaira K, Hokari R, Tsuzuki Y, Okada Y, Komoto S, Watanabe C, Kurihara C, Kawaguchi A, Nagao S, Azuma M, et al: Blockade of B7-H1 or B7-DC induces an anti-tumor effect in a mouse pancreatic cancer model. Int J Oncol 35: 741-749, 2009.

17. Brahmer JR, Drake CG, Wollner I, Powderly JD, Picus J, Sharfman WH, Stankevich E, Pons A, Salay TM, McMiller TL, et al: Phase I study of single-agent anti-programmed death-1 (MDX-1106) in refractory solid tumors: Safety, clinical activity, pharmacodynamics, and immunologic correlates. J Clin Oncol 28: 3167-3175, 2010.

18. Brahmer JR, Tykodi SS, Chow LQ, Hwu WJ, Topalian SL, Hwu P, Drake CG, Camacho LH, Kauh J, Odunsi K, et al: Safety and activity of anti-PD-L1 antibody in patients with advanced cancer. N Engl J Med 366: 2455-2465, 2012.

19. Le DT, Lutz E, Uram JN, Sugar EA, Onners B, Solt S, Zheng L, Diaz LA Jr, Donehower RC, Jaffee EM, et al: Evaluation of ipilimumab in combination with allogeneic pancreatic tumor cells transfected with a GM-CSF gene in previously treated pancreatic cancer. J Immunother 36: 382-389, 2013.

20. Lutz ER, Wu AA, Bigelow E, Sharma R, Mo G, Soares K, Solt S, Dorman A, Wamwea A, Yager A, et al: Immunotherapy converts nonimmunogenic pancreatic tumors into immunogenic foci of immune regulation. Cancer Immunol Res 2: 616-631, 2014.

21. Zhang P, Su DM, Liang M and Fu J: Chemopreventive agents induce programmed death-1-ligand 1 (PD-L1) surface expression in breast cancer cells and promote PD-L1-mediated T cell apoptosis. Mol Immunol 45: 1470-1476, 2008.

22. Ghebeh H, Lehe C, Barhoush E, Al-Romaih K, Tulbah A, Al-Alwan M, Hendrayani SF, Manogaran P, Alaiya A, Al-Tweigeri T, et al: Doxorubicin downregulates cell surface B7-H1 expression and upregulates its nuclear expression in breast cancer cells: Role of B7-H1 as an anti-apoptotic molecule. Breast Cancer Res 12: R48, 2010.

23. Gong W, Song Q, Lu X, Gong W, Zhao J, Min P and Yi X: Paclitaxel induced B7-H1 expression in cancer cells via the MAPK pathway. J Chemother 23: 295-299, 2011.

24. Peng J, Hamanishi J, Matsumura N, Abiko K, Murat K, Baba T, Yamaguchi K, Horikawa N, Hosoe Y, Murphy SK, et al: Chemotherapy induces programmed cell death-ligand 1 overexpression via the nuclear factor- $\kappa \mathrm{B}$ to foster an immunosuppressive tumor microenvironment in ovarian cancer. Cancer Res 75: 5034-5045, 2015.

25. Higashimura $Y$, Naito $Y$, Takagi T, Mizushima K, Hirai Y, Harusato A, Ohnogi $\mathrm{H}$, Yamaji R, Inui $\mathrm{H}$, Nakano $\mathrm{Y}$, et al: Oligosaccharides from agar inhibit murine intestinal inflammation through the induction of heme oxygenase-1 expression. J Gastroenterol 48: 897-909, 2013.

26. Kanda Y: Investigation of the freely available easy-to-use software 'EZR' for medical statistics. Bone Marrow Transplant 48: 452-458, 2013.

27. Lee SJ, Jang BC, Lee SW, Yang YI, Suh SI, Park YM, Oh S, Shin JG, Yao S, Chen L, et al: Interferon regulatory factor-1 is prerequisite to the constitutive expression and IFN-gammainduced upregulation of B7-H1 (CD274). FEBS Lett 580: $755-762,2006$.

28. Mimura K, Kua LF, Shiraishi K, Kee Siang L, Shabbir A, Komachi M, Suzuki Y, Nakano T, Yong WP, So J, et al: Inhibition of mitogen-activated protein kinase pathway can induce upregulation of human leukocyte antigen class I without PD-L1-upregulation in contrast to interferon- $\gamma$ treatment. Cancer Sci 105: 1236-1244, 2014 
29. Lee SK, Seo SH, Kim BS, Kim CD, Lee JH, Kang JS, Maeng PJ and Lim JS: IFN-gamma regulates the expression of B7-H1 in dermal fibroblast cells. J Dermatol Sci 40: 95-103, 2005.

30. Isomura I, Shintani Y, Yasuda Y, Tsujimura K and Morita A: Induction of regulatory dendritic cells by topical application of NF-kappaB decoy oligodeoxynucleotides. Immunol Lett 119: 49-56, 2008.

31. Qin X, Liu C,Zhou Y and Wang G: Cisplatin induces programmed death-1-ligand 1(PD-L1) over-expression in hepatoma H22 cells via Erk/MAPK signaling pathway. Cell Mol Biol (Suppl 56): OL1366-OL1372, 2010.

32. Maude SL, Frey N, Shaw PA, Aplenc R, Barrett DM, Bunin NJ, Chew A, Gonzalez VE, Zheng Z, Lacey SF, et al: Chimeric antigen receptor $\mathrm{T}$ cells for sustained remissions in leukemia. $\mathrm{N}$ Engl J Med 371: 1507-1517, 2014.

33. Bocci G, Danesi R, Di Paolo AD, Innocenti F, Allegrini G, Falcone A, Melosi A, Battistoni M, Barsanti G, Conte PF, et al: Comparative pharmacokinetic analysis of 5-fluorouracil and its major metabolite 5-fluoro-5,6-dihydrouracil after conventional and reduced test dose in cancer patients. Clin Cancer Res 6: 3032-3037, 2000.

34. Kroep JR, Giaccone G, Voorn DA, Smit EF, Beijnen JH, Rosing $\mathrm{H}$, van Moorsel CJ, van Groeningen CJ, Postmus PE, Pinedo HM, et al: Gemcitabine and paclitaxel: Pharmacokinetic and pharmacodynamic interactions in patients with non-smallcell lung cancer. J Clin Oncol 17: 2190-2197, 1999.

35. Sakai H, Kokura S, Ishikawa T, Tsuchiya R, Okajima M, Matsuyama T, Adachi S, Katada K, Kamada K, Uchiyama K, et al: Effects of anticancer agents on cell viability, proliferative activity and cytokine production of peripheral blood mononuclear cells. J Clin Biochem Nutr 52: 64-71, 2013.

36. Okino H, Maeyama R, Manabe T, Matsuda T and Tanaka M: Trans-tissue, sustained release of gemcitabine from photocured gelatin gel inhibits the growth of heterotopic human pancreatic tumor in nude mice. Clin Cancer Res 9: 5786-5793, 2003.

37. Pardoll DM: The blockade of immune checkpoints in cancer immunotherapy. Nat Rev Cancer 12: 252-264, 2012.
38. Parsa AT, Waldron JS, Panner A, Crane CA, Parney IF, Barry JJ, Cachola KE, Murray JC, Tihan T, Jensen MC, et al: Loss of tumor suppressor PTEN function increases B7-H1 expression and immunoresistance in glioma. Nat Med 13: 84-88, 2007.

39. Marzec M, Zhang Q, Goradia A, Raghunath PN, Liu X, Paessler M, Wang HY, Wysocka M, Cheng M, Ruggeri BA, et al: Oncogenic kinase NPM/ALK induces through STAT3 expression of immunosuppressive protein CD274 (PD-L1, B7-H1). Proc Natl Acad Sci USA 105: 20852-20857, 2008.

40. Deer EL, González-Hernández J, Coursen JD, Shea JE, Ngatia J, Scaife CL, Firpo MA and Mulvihill SJ: Phenotype and genotype of pancreatic cancer cell lines. Pancreas 39: 425-435, 2010.

41. Awasthi N, Zhang C, Schwarz AM, Hinz S, Wang C, Williams NS, Schwarz MA and Schwarz RE: Comparative benefits of Nab-paclitaxel over gemcitabine or polysorbate-based docetaxel in experimental pancreatic cancer. Carcinogenesis 34: 2361-2369, 2013

42. Holcomb B, Yip-Schneider MT, Matos JM, Dixon J, Kennard J, Mahomed J, Shanmugam R, Sebolt-Leopold J and Schmidt CM: Pancreatic cancer cell genetics and signaling response to treatment correlate with efficacy of gemcitabine-based molecular targeting strategies. J Gastrointest Surg 12: 288-296, 2008.

43. Adachi S, Kokura S, Okayama T, Ishikawa T, Takagi T, Handa O, Naito Y and Yoshikawa T: Effect of hyperthermia combined with gemcitabine on apoptotic cell death in cultured human pancreatic cancer cell lines. Int J Hyperthermia 25: 210-219, 2009.

44. Cao LP, Song JL, Yi XP and Li YX: Double inhibition of NF- $\kappa B$ and XIAP via RNAi enhances the sensitivity of pancreatic cancer cells to gemcitabine. Oncol Rep 29: 1659-1665, 2013.

45. Thoennissen NH, Iwanski GB, Doan NB, Okamoto R, Lin P, Abbassi S, Song JH, Yin D, Toh M, Xie WD, et al: Cucurbitacin B induces apoptosis by inhibition of the JAK/STAT pathway and potentiates antiproliferative effects of gemcitabine on pancreatic cancer cells. Cancer Res 69: 5876-5884, 2009. 